\title{
Minimally invasive instrumentation without fusion during posterior thoracic corpectomies: a comparison of percutaneously instrumented nonfused segments with open instrumented fused segments
}

\author{
Darryl Lau, MD, and Dean Chou, MD \\ Department of Neurological Surgery, University of California, San Francisco, California
}

\begin{abstract}
OBJECTIVE During the mini-open posterior corpectomy, percutaneous instrumentation without fusion is performed above and below the corpectomy level. In this study, the authors' goal was to compare the perioperative and long-term implant failure rates of patients who underwent nonfused percutaneous instrumentation with those of patients who underwent traditional open instrumented fusion.
\end{abstract}

METHODS Adult patients who underwent posterior thoracic corpectomies with cage reconstruction between 2009 and 2014 were identified. Patients who underwent mini-open corpectomy had percutaneous instrumentation without fusion, and patients who underwent open corpectomy had instrumented fusion above and below the corpectomy site. The authors compared perioperative outcomes and rates of implant failure requiring reoperation between the open (fused) and mini-open (unfused) groups.

RESULTS A total of 75 patients were identified, and 53 patients ( 32 open and 21 mini-open) were available for followup. The mean patient age was 52.8 years, and $56.6 \%$ of patients were male. There were no significant differences in baseline variables between the 2 groups. The overall perioperative complication rate was $15.1 \%$, and there was no significant difference between the open and mini-open groups ( $18.8 \%$ vs $9.5 \% ; p=0.359)$. The mean hospital stay was 10.5 days. The open group required a significantly longer stay than the mini-open group (12.8 vs 7.1 days; $p<0.001)$. Overall implant failure rates requiring reoperation were $1.9 \%$ at 6 months, $9.1 \%$ at 1 year, and $14.7 \%$ at 2 years. There were no significant differences in reoperation rates between the open and mini-open groups at 6 months $(3.1 \%$ vs $0.0 \%$, $p=0.413), 1$ year $(10.7 \%$ vs $6.2 \%, p=0.620)$, and 2 years $(18.2 \%$ vs $8.3 \%, p=0.438)$. The overall mean follow-up was 29.2 months.

CONCLUSIONS These findings suggest that percutaneous instrumentation without fusion in mini-open transpedicular corpectomies offers similar implant failure and reoperation rates as open instrumented fusion as far out as 2 years of follow-up.

https://thejns.org/doi/abs/10.3171/2016.12.SPINE16598

KEY WORDS corpectomy; costotransversectomy; expandable cage; fusion; minimally invasive; mini-open; percutaneous; transpedicular corpectomy; thoracic

$\mathrm{O}$ VER the last decade, minimally invasive surgery (MIS) and mini-open approaches to the spine have shown outcomes comparable to those of traditional open approaches in the treatment of many spinal disorders. ${ }^{6,11,13,24,25,27}$ Additionally, MIS approaches have been associated with less intraoperative blood loss, less postoperative pain, shorter hospital stays, and quicker recoveries; there is even evidence that these advantages continue to benefit patients at $1-2$ years after surgery. ${ }^{5,13,14,21}$ The majority of studies evaluating the utility and efficacy of MIS techniques concentrate on simple decompression and lumbar fusions of degenerative etiology. Less frequently, MIS and mini-open approaches have been used to treat spinal tumors, osteomyelitis, sagittal deformities, and scoliosis. ${ }^{13,24-27}$ The surgical management of such diseases can be technically challenging, even with traditional open ap-

ABBREVIATIONS ASIA = American Spinal Injury Association; MIS = minimally invasive surgery.

SUBMITTED May 22, 2016. ACCEPTED December 5, 2016.

INCLUDE WHEN CITING Published online April 21, 2017; DOI: 10.3171/2016.12.SPINE16598. 
proaches because complex spinal reconstruction is usually required.

There are technical limitations and challenges when MIS approaches are used for spine surgery that require complex reconstruction, cage placement, and long-segment posterior fixation. ${ }^{10,28}$ First, access to treat fixed spinal deformities and destructive disease processes (tumors and infection) can be limited through MIS access ports. Additionally, there is often loss of normal anatomical landmarks, further making it even more challenging when the surgeon is only able to gain a limited view of the spine through small incisions and tubular retractors. However, this can be partially overcome with mini-open approaches in which a normal fascial opening and exposure is performed over the site of osteotomy or vertebral column resection. Percutaneous fixation above and below the pathology can be performed for spinal stabilization. ${ }^{2,3,13,19,20,26}$ Another major technical challenge to MIS techniques is the limited ability to perform extensive and robust arthrodesis to promote bony fusion during percutaneous instrumentation. ${ }^{6,23}$

Therefore, there is appropriate clinical concern and uncertainty as to the longevity of performing complex spinal reconstruction (3-column surgery) with percutaneous instrumentation without fusion. This study seeks to answer the question of whether percutaneous instrumentation without fusion during mini-open corpectomy is associated with higher rates of implant failure requiring reoperation compared with traditional open corpectomy with complete instrumented fusion.

\section{Methods}

The University of California, San Francisco, Committee of Human Research formally approved this study.

\section{Patients}

A consecutive cohort of adult patients who underwent either open or mini-open posterior thoracic corpectomy with cage reconstruction was identified. All surgeries were performed by the senior author (D.C.). The study period of interest spanned from 2009 to 2014. Inclusion criteria were patients 18 years or older who underwent a complete posterior transpedicular or costotransversectomy thoracic corpectomy with expandable cage reconstruction. All patients underwent complete corpectomy with nearly $100 \%$ vertebral body resection to allow an expandable cage to be placed all the way anteriorly along the epiphyseal ring for reconstruction of the anterior column. Treated pathologies included vertebral body fractures, osteomyelitis, and tumors. For infections and tumors, the diagnosis was confirmed through histopathological analysis of intraoperative specimens. The open corpectomy was performed through a traditional exposure of the spine, open pedicle screw instrumentation, and arthrodesis along the entire length of the construct with either allograft or autograft. The mini-open corpectomy was performed with a midline skin incision and opening of the fascia only at the level of corpectomy. The fascia was left intact over pedicle screw instrumentation sites. Percutaneous pedicle screw placement was performed above and below the site of corpec- tomy through the fascia using MIS techniques (Fig. 1). ${ }^{13}$ No bony preparation, exposure of the bone, or decortication was performed in mini-open cases over the percutaneous instrumented sites. Fusion was performed only over the corpectomy site where the fascia was open. Local autograft, iliac crest graft, or allograft was used only over the corpectomy site in the mini-open corpectomies. Fusion with local autograft, iliac crest graft, or allograft was performed at the corpectomy site and along the entire length of the construct in open corpectomies. No bone morphogenetic protein or cell-based allograft was used in the study cohort. Spinal constructs consisted of pedicle screw instrumentation 2 or 3 levels above and below the corpectomy site (even in the presence of multilevel consecutive corpectomies), and an expandable titanium cage filled with autograft or allograft was used to reconstruct the anterior column.

\section{Selection for Open Versus Mini-Open Transpedicular Corpectomy}

Patients underwent either open or mini-open surgery based on operating room staff and availability of equipment. For instance, if surgery could be started during normal work hours, a mini-open approach was performed, even if the case was an emergency. However, if surgery had to be done outside normal work hours (nights) or on weekends when regular spine operating room personnel, MIS screw systems, or navigation support was not available, the traditional open procedure was performed. The senior surgeon (D.C.) performed all operations in this study, regardless of the time of surgery.

\section{Data Points}

A detailed retrospective review of the medical records was performed. Data related to patient demographics, diagnosis, surgery, and outcomes were collected. Demographic and preoperative data points of interest included age, sex, weight (measured in kilograms), diagnosis, and

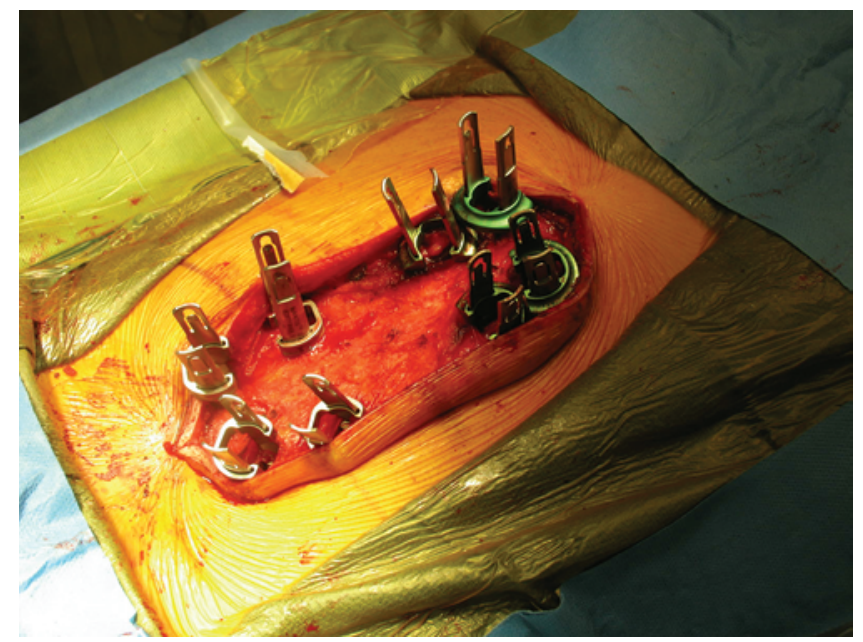

FIG. 1. Intraoperative photograph demonstrating pedicle screw fixation without arthrodesis. A midline incision is made to expose the overlying fascia. The fascia is kept intact and pedicle fixation is performed with percutaneous instrumentation. No arthrodesis is performed at the levels of pedicle fixation. Figure is available in color online only. 
neurological status (measured using the American Spinal Injury Association [ASIA] Impairment Scale). ${ }^{1}$ The number of corpectomies performed was categorized as 1,2, and 3 or more levels. Perioperative outcomes of interest were intraoperative blood loss, perioperative complications, and length of hospital stay (days from date of surgery to date of discharge). Complications were defined as any unforeseen event related to surgery that required additional medical and/or surgical intervention. With regard to long-term outcomes, outcomes of interest were complications after discharge and implant failure requiring revision surgery. Implant failure was defined as any implant-related issues that resulted in radiographic implant loosening, breakage, dislodgment, spinal instability, and/or progressive deformity of the instrumented segments requiring surgical intervention.

\section{Statistical Analysis}

The general cohort was first stratified into 2 groups: patients who underwent either open corpectomy with complete fusion or mini-open corpectomy with nonfused percutaneous instrumentation. Comparisons of preoperative, perioperative, and follow-up outcomes were performed. For categorical outcomes, the Fisher exact test was used. For continuous outcomes, a 2-tailed Student t-test was used. For this study, $\mathrm{p}<0.050$ was used as a threshold for statistical significance. All statistical analysis was performed using SAS software (version 9.4, SAS Institute).

\section{Results}

Over the 5-year period, a total of 75 patients underwent posterior transpedicular or costotransversectomy corpectomy with cage reconstruction. Twenty patients had died (a majority because of cancer/metastasis) with less than 6 months follow-up and 2 patients were lost to follow-up: 13 of 45 patients in the open group (28.9\%) and 9 of 30 patients in the mini-open group (30.0\%). This resulted in a total of 53 patients for final analysis: 32 in the open group and 21 in the mini-open group. There were no significant differences in demographics, diagnosis, and preoperative clinical baselines between the open and mini-open group (Table 1). The overall mean age was 52.8 years, and $56.6 \%$ of patients were male. The distributions of diagnosis were tumors in $64.2 \%$, osteomyelitis in $24.5 \%$, and vertebral body fractures in $11.3 \%$ of patients. With regard to preoperative neurological status, the majority of patients had ASIA grades of either E (37.7\%) or D (45.3\%). The remaining distributions were ASIA Grades $\mathrm{C}$ in $13.2 \%$, B in $1.9 \%$, and $\mathrm{A}$ in $1.9 \%$. Of the 53 patients, $64.2 \%$ underwent a 1-level corpectomy, $26.4 \%$ underwent a 2 -level corpectomy, and $9.4 \%$ underwent a corpectomy of 3 or more levels. The distribution of levels at which corpectomies were performed can be seen in Table 1 .

Table 2 compares perioperative outcomes between patients who underwent either open corpectomy with complete fusion or mini-open corpectomy with percutaneous instrumentation without fusion. The overall mean blood loss was $1618.4 \mathrm{ml}$. Patients who underwent open corpectomies had significantly greater amount of blood loss than patients who underwent mini-open corpectomies (1925.8
TABLE 1. Demographics, diagnosis, and preoperative clinical baseline in patients who underwent either open corpectomy with instrumented fusion or mini-open corpectomy with nonfused percutaneous instrumentation

\begin{tabular}{|c|c|c|c|c|}
\hline Variable & $\begin{array}{l}\text { Overall } \\
(n=53)\end{array}$ & $\begin{array}{c}\text { Open } \\
(n=32)\end{array}$ & $\begin{array}{l}\text { Mini-Open } \\
(n=21)\end{array}$ & $\begin{array}{c}p \\
\text { Value }\end{array}$ \\
\hline Mean age (yrs) & $52.8 \pm 14.8$ & $54.4 \pm 14.3$ & $50.3 \pm 15.7$ & 0.331 \\
\hline Male sex & $30(56.6)$ & $17(53.1)$ & $13(61.9)$ & 0.528 \\
\hline Mean weight $(\mathrm{kg})$ & $72.7 \pm 17.6$ & $70.8 \pm 15.7$ & $75.6 \pm 20.4$ & 0.346 \\
\hline Diagnosis & & & & 0.932 \\
\hline Tumor & $34(64.2)$ & $20(62.5)$ & $14(66.7)$ & \\
\hline Osteomyelitis & $13(24.5)$ & $8(25.0)$ & $5(23.8)$ & \\
\hline Trauma & $6(11.3)$ & $4(12.5)$ & $2(9.5)$ & \\
\hline ASIA grade & & & & 0.744 \\
\hline$E$ & $20(37.7)$ & $11(34.4)$ & $9(42.9)$ & \\
\hline$D$ & $24(45.3)$ & $14(43.8)$ & $10(47.6)$ & \\
\hline C & $7(13.2)$ & $5(15.6)$ & $2(9.5)$ & \\
\hline B & $1(1.9)$ & $1(3.1)$ & $0(0.0)$ & \\
\hline$A$ & $1(1.9)$ & $1(3.1)$ & $0(0.0)$ & \\
\hline No. of corpectomies & & & & 0.446 \\
\hline 1 & $34(64.2)$ & $20(62.5)$ & $14(66.7)$ & \\
\hline 2 & $14(26.4)$ & $10(31.2)$ & $4(19.0)$ & \\
\hline$\geq 3$ & $5(9.4)$ & $2(6.2)$ & $3(14.3)$ & \\
\hline \multicolumn{5}{|l|}{ Corpectomy level ${ }^{*}$} \\
\hline $\mathrm{T}-2$ & $5(9.4)$ & $5(15.6)$ & $0(0.0)$ & \\
\hline T-3 & $6(11.3)$ & $5(15.6)$ & $1(4.8)$ & \\
\hline $\mathrm{T}-4$ & $7(13.2)$ & $4(12.5)$ & $3(14.3)$ & \\
\hline $\mathrm{T}-5$ & $4(7.5)$ & $1(3.1)$ & $3(14.3)$ & \\
\hline T-6 & $5(9.4)$ & $1(3.1)$ & $4(19.0)$ & \\
\hline $\mathrm{T}-7$ & $4(7.5)$ & $2(6.2)$ & $2(9.5)$ & \\
\hline $\mathrm{T}-8$ & $4(7.5)$ & $3(9.4)$ & $1(4.8)$ & \\
\hline T-9 & $4(7.5)$ & $2(6.2)$ & $2(9.5)$ & \\
\hline $\mathrm{T}-10$ & $2(3.8)$ & $2(6.2)$ & $0(0.0)$ & \\
\hline $\mathrm{T}-11$ & $2(3.8)$ & $2(6.2)$ & $0(0.0)$ & \\
\hline $\mathrm{T}-12$ & $10(18.9)$ & $5(15.6)$ & $5(23.8)$ & \\
\hline
\end{tabular}

Values are presented as the number of patients (\%) unless otherwise indicated. Mean values are reported as the mean \pm SD.

* In patients who underwent a corpectomy of 2 or more levels, their most rostral vertebral level is represented on the table.

vs $1150.0, p=0.035)$. The overall perioperative complication rate was $15.1 \%$. The open group (18.8\%) experienced a higher rate of complications than the mini-open group $(9.5 \%)$, but this was not statistically significant $(\mathrm{p}=0.359)$. The types of complications encountered in the open group were epidural hematomas requiring intervention ( 3 patients), coagulopathy requiring aggressive resuscitation (1 patient), wound dehiscence (1 patient), and pleural effusion requiring chest tube placement (1 patient). In the mini-open group, 2 patients developed wound infections. The overall length of hospital stay was 10.5 days. The open group had a significantly longer hospital stay than the mini-open group (12.8 days vs 7.1 days, $\mathrm{p}<0.001$ ).

The primary goal of the study was to evaluate the outcomes of patients who underwent mini-open corpectomy 
TABLE 2. Comparison of perioperative outcomes

\begin{tabular}{lcccc}
\hline \multicolumn{1}{c}{ Variable } & Overall $(n=53)$ & Open $(n=32)$ & Mini-Open $(n=21)$ & $p$ Value \\
\hline Mean blood loss $(\mathrm{ml})$ & $1618.4 \pm 1319.1$ & $1925.8 \pm 1530.1$ & $1150.0 \pm 716.6$ & 0.035 \\
\hline No. of periop complications (\%) & $8(15.1)$ & $6(18.8)$ & $2(9.5)$ & 0.359 \\
\hline Mean length of stay (days) & $10.5 \pm 6.3$ & $12.8 \pm 6.8$ & $7.1 \pm 3.5$ & $<0.001$ \\
\hline
\end{tabular}

without fusion over the percutaneous sites and compare its durability to traditional open corpectomy with complete instrumented fusion. These results can be seen in Table 3. The overall mean follow-up for the cohort was 29.2 months: 30.9 months in the open group and 26.5 months in the mini-open group $(\mathrm{p}=0.382)$. The overall long-term complication rate was $15.1 \%$, and there was no significant difference between the 2 groups $(18.8 \%$ vs $9.5 \%)(\mathrm{p}=0.359)$. Implant failure rates were examined at 6-, 12-, and 24-month intervals. Overall implant failure rates were $1.9 \%$ at 6 months, $9.1 \%$ at 12 months, and $14.7 \%$ at 24 months. Implant failure rates were similar between the open and the mini-open groups at 6 months $(3.1 \%$ vs $0.0 \%, \mathrm{p}=0.413), 12$ months $(10.7 \%$ vs $6.2 \%, \mathrm{p}=0.620)$, and 24 months $(18.2 \%$ vs $8.3 \%, \mathrm{p}=0.438)$ (Fig. 2). At the most recent follow-up, a similar proportion of patients improved or remained neurologically normal when comparing the 2 groups. There was no significant difference in the distribution of ASIA scores between the 2 groups ( $p$ $=0.193$ ). The ASIA grade distribution in the open group was E (78.1\%), D (15.6\%), and B (6.2\%). In the mini-open group, the distribution was ASIA Grade E in $66.7 \%$ and Grade D in $33.3 \%$.

\section{Discussion}

Over the past decade there has been an accumulation of evidence that MIS spine surgery for simple decompression and short-segment fusions is able to offer less intraoperative blood loss, reduced postoperative pain, shorter hospital stays, and comparable outcomes to open surgery at 1 to 2 years after surgery. ${ }^{5,13,14,21}$ However, there are inherent drawbacks when using MIS percutaneous techniques for spinal instrumentation. One of the main concerns is the inability to perform adequate arthrodesis and fusion at percutaneously instrumented levels in the absence of an interbody device, thus theoretically placing patients at higher risk of implant failure. This concept is especially relevant as MIS techniques are being used more readily to take on complex spinal pathologies that require vertebral column resection, long-segment instrumentation, and spinal column reconstruction. ${ }^{2,3,13,24,27}$ Understanding the ramifications of percutaneous instrumentation without fusion is important in the long-term clinical management of patients. Additionally, this is especially relevant in patients with metastatic spinal tumors who have limited life expectancy and who would benefit from less-morbid mini-open approaches to maintain a good quality of life.

The most robust published data pertaining to pedicle screw instrumentation without fusion stem from the spinal trauma literature. More specifically, instrumentation without fusion in the surgical management of thoracolumbar burst fractures has been thoroughly evaluated. Wang et al. performed a randomized prospective clinical trial comparing open short-segment instrumentation with or without fusion. ${ }^{29}$ With a mean follow-up of 42 months, they reported an overall implant failure rate of $13.8 \%$, and there was no significant difference in the implant failure rate between patients who underwent fusion and no fusion $(16.7 \%$ vs $10.7 \%)$. Similarly, Dai et al. performed a prospective randomized trial comparing open short-segment instrumentation with or without fusion for thoracolumbar (T11-L2) superior endplate burst fractures. ${ }^{4}$ With a 5-year minimum follow-up, no patient in either group required reoperation for implant failure, suggesting that instrumentation without fusion offers comparable results to instrumentation with fusion in certain clinical settings.

TABLE 3. Comparison of long-term clinical outcomes and implant failure rates

\begin{tabular}{lcccc}
\hline \multicolumn{1}{c}{ Variable } & Overall $(n=53)$ & Open $(n=32)$ & Mini-Open $(n=21)$ & $p$ Value \\
\hline Mean follow-up (mos) & $29.2 \pm 17.9$ & $30.9 \pm 18.4$ & $26.5 \pm 17.1$ & 0.382 \\
\hline Follow-up complications & $8(15.1)$ & $6(18.8)$ & $2(9.5)$ & 0.359 \\
\hline Implant failure & & & $0 / 21(0.0)$ & 0.413 \\
\hline 6 mos & $1 / 53(1.9)$ & $1 / 32(3.1)$ & $1 / 16(6.2)$ & 0.620 \\
\hline 12 mos & $4 / 44(9.1)$ & $3 / 28(10.7)$ & $1 / 12(8.3)$ & 0.438 \\
\hline 24 mos & $5 / 34(14.7)$ & $4 / 22(18.2)$ & & 0.193 \\
\hline ASIA grade & & & $14(66.7)$ & $7(33.3)$ \\
\hline E & $39(73.6)$ & $25(78.1)$ & $0(0.0)$ & \\
\hline D & $12(22.6)$ & $5(15.6)$ & $0(0.0)$ & \\
\hline C & $0(0.0)$ & $0(0.0)$ & $0(0.0)$ & \\
\hline B & $2(3.8)$ & $2(6.2)$ & & \\
\hline A & $0(0.0)$ & $0(0.0)$ & &
\end{tabular}

Values are presented as the number of patients (\%) unless otherwise indicated. 


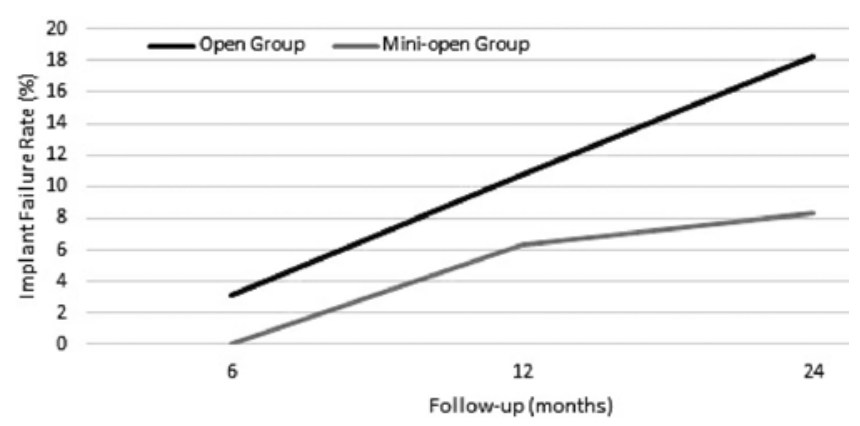

FIG. 2. Comparison of implant failure rates in patients who underwent open versus mini-open corpectomy. Implant failure rates were examined at 6-, 12-, and 24-month intervals. Implant failure rates were similar between the open and the mini-open group at 6 months (3.1\% vs $0.0 \%, p=$ $0.413), 12$ months $(10.7 \%$ vs $6.2 \%, p=0.620)$, and 24 months $(18.2 \%$ vs $8.3 \%, p=0.438)$.

With regard to long-term outcomes and MIS instrumentation, however, there is a paucity of data comparing traditional open instrumented fusion and percutaneous pedicle fixation without fusion. One study by Lee et al. examined follow-up outcomes at 3 months and 6 months in trauma patients and found similar rates of instrumentation failure between the percutaneous nonfused group (3.1\%) and the open fused group (3.7\%). ${ }^{16}$ These preliminary results are promising and support the use of percutaneous instrumentation without fusion. However, there still is a lack of data regarding long-term implant failure rates, and there are no studies evaluating the use of percutaneous pedicle fixation without fusion in the setting of long-segment instrumentation with 3-column surgeries such as corpectomies.

Pseudarthrosis and implant failure rates requiring reoperation following open thoracic corpectomy with cage reconstruction are generally low, with reported rates ranging from $0 \%$ to $10 \% .^{7-9,15,22}$ These failure rates are likely dependent on the time point of follow-up, as we have demonstrated higher rates of implant failure with longer follow-up. Nonetheless, in our study, the implant failure rates following mini-open corpectomy and percutaneous instrumentation without fusion at 6,12 , and 24 months $(0.0 \%, 6.2 \%$, and $8.3 \%$, respectively) fall within the range of previously reported implant failure rates after open surgery with fusion. This finding suggests that fusion at the corpectomy site alone with supplemental percutaneous instrumentation may offer comparable durability to open complete fusion of the entire construct.

Possible explanations for comparable durability may be related to the MIS technique itself, the normal anatomical biomechanics of the thoracic spine, and use of a cage. With MIS percutaneous pedicle instrumentation, there is inherent preservation of the paraspinal muscles and the posterior ligamentous complex, which normally provides significant mechanical support of the spine. ${ }^{12,17}$ These paraspinal muscles and ligaments are often destroyed or stripped during open procedures. Furthermore, there may be an anatomical uniqueness to different regions of the spine that could account for the similar implant failure rate when performing corpectomy with nonfused percutaneous instrumentation compared with open instrumented fusion. In this study, all corpectomies were performed in the thoracic spine, and the majority of instrumentation involved the thoracic spine and thoracolumbar junction. Compared with the lumbar spine, the thoracic spine intrinsically has less motion and increases stability due to rib cage support and less facet cartilage. This could result in auto-arthrodesis of the facets after instrumentation. Alternatively, it may be that the thoracic spine is naturally at lower risk of implant failure due to less normal motion, even in the absence of auto-arthrodesis of facets.

Another consideration of this study is that although we evaluated the adjacent nonfused segments next to the corpectomy site, it must be emphasized that not only was the corpectomy site fused (in both open and mini-open approaches), but also an interbody cage was always used in all cases. In addition, to arthrodesis at the corpectomy site, cage placement offers a solid anterior structural support which helps takes on a large portion of the spinal load. Thus, the question this study addresses is not outcomes related generically to instrumentation of the spine without fusion, but specifically instrumentation with or without fusion adjacent to a fused segment with anterior column support. It is important to recognize that this is different from simple spinal instrumentation without fusion in the absence of anterior column reconstruction for a degenerative lumbar condition. Thus, it would be critical not to directly translate these results to degenerative conditions of the lumbar spine.

Although our study focused on MIS percutaneous instrumentation without fusion, there are techniques that allow for facet arthrodesis at the instrumented levels with tubular retractors. After cannulation of the pedicles, facets can be decorticated and graft can be inserted into the facet joint to promote fusion. ${ }^{26}$ This type of facet joint fusion has been shown to provide similar fusion rates to open posterior lateral fusion for lumbar spondylolistheisis. ${ }^{18} \mathrm{In}$ addition, this focused facet fusion technique has been used during mini-open pedicle subtraction osteotomy with long-segment thoracolumbar percutaneous instrumentation for deformity correction and has demonstrated at least an $80 \%$ solid fusion rate on follow-up CT imaging. ${ }^{26}$

This study is subjected to limitations inherent to its retrospective design and the novelty of the surgical technique. Specifically, the patient cohort is at risk for selection biases and confounders. For example, slightly more mini-open cases were performed later in the series when experience with the mini-open technique was greater; this may have had an impact on the patient selection as to who received a mini-open procedure and who received an open procedure. Nonetheless, the influence of this confounder is likely minimal because the distribution of cases in each group spans similar time periods. In addition, the selection bias was based on a temporal criterion (more mini-open procedures with more experience) rather than individual patient-specific criteria (frail or sick patients vs robust and healthy patients). Additionally, while we demonstrate no significant differences in major baseline and clinical variables between the groups, there are likely other factors not measured that may impact implant failure rates, some of which include bone quality or pathological bony destruction from infection and tumors at fused levels. A prospec- 
tive study or clinical trial would be better able to address these confounders by establishing explicit exclusion-inclusion criteria and performing standard tests at specific time points. Next, the relatively small cohort sizes and loss to follow-up at various time points limits the power of the study findings. Additionally, the lack of degenerative spine pathologies confines broad generalization of these data. Thus, applying instrumentation without fusion for degenerative cases would be ill advised based solely upon these study findings. Lastly, there may be drawbacks of using implant failure and revision surgery as a proxy for fusion rates instead of CT imaging. Although obtaining fine-cut CT imaging through the instrumented areas would be ideal, it was simply not practical given the costs associated with the test and lack of indication in the mini-open cohort (given that no bony fusion was attempted).

\section{Conclusions}

The findings from this study suggest that the rates of implant failure-requiring reoperation in patients who underwent either open corpectomy with complete instrumented fusion or mini-open corpectomy with unfused percutaneous instrumentation were comparable. Specifically, failure rates of instrumentation with or without fusion were similar at 6, 12, and 24 months. Additional larger studies are warranted, but unfused percutaneous instrumentation should be considered as a viable option in the appropriate clinical setting. Particularly, patients with short life expectancies (spinal metastasis) or those too ill to undergo open instrumented fusion may benefit from this technique.

\section{References}

1. American Spinal Injury Association: International Standards for Neurological Classifications of Spinal Cord Injury, revised. Chicago: American Spinal Injury Association, 2000

2. Charles YP, Schuller S, Sfeir G, Steib JP: Mini open tumor resection and percutaneous instrumentation for T11 renal cell carcinoma metastasis. Eur Spine J 22:1440-1442, 2013

3. Chou D, Lau D, Roy E: Feasibility of the mini-open vertebral column resection for severe thoracic kyphosis. J Clin Neurosci 21:841-845, 2014

4. Dai LY, Jiang LS, Jiang SD: Posterior short-segment fixation with or without fusion for thoracolumbar burst fractures. A five to seven-year prospective randomized study. J Bone Joint Surg Am 91:1033-1041, 2009

5. Djurasovic M, Rouben DP, Glassman SD, Casnellie MT, Carreon LY: Clinical outcomes of minimally invasive versus open TLIF: a propensity-matched cohort study. Am J Orthop 45:E77-E82, 2016

6. Hamilton DK, Kanter AS, Bolinger BD, Mundis GM Jr, Nguyen S, Mummaneni PV, et al: Reoperation rates in minimally invasive, hybrid and open surgical treatment for adult spinal deformity with minimum 2-year follow-up. Eur Spine J 25:2605-2611, 2016

7. Hofstetter CP, Chou D, Newman CB, Aryan HE, Girardi FP, Härtl R: Posterior approach for thoracolumbar corpectomies with expandable cage placement and circumferential arthrodesis: a multicenter case series of 67 patients. J Neurosurg Spine 14:388-397, 2011

8. Holland CM, Bass DI, Gary MF, Howard BM, Refai D: Thoracic lateral extracavitary corpectomy for anterior column reconstruction with expandable and static titanium cages: clinical outcomes and surgical considerations in a consecutive case series. Clin Neurol Neurosurg 129:37-43, 2015

9. Joubert C, Adetchessi T, Peltier E, Graillon T, Dufour H, Blondel B, et al: Corpectomy and vertebral body reconstruction with expandable cage placement and osteosynthesis via the single stage posterior approach: a retrospective series of 34 patients with thoracic and lumbar spine vertebral body tumors. World Neurosurg 84:1412-1422, 2015

10. Kanter AS, Tempel ZJ, Ozpinar A, Okonkwo DO: A review of minimally invasive procedures for the treatment of adult spinal deformity. Spine (Phila Pa 1976) 41 (Suppl 8):S59S65, 2016

11. Khan NR, Clark AJ, Lee SL, Venable GT, Rossi NB, Foley KT: Surgical outcomes for minimally invasive vs open transforaminal lumbar interbody fusion: an updated systematic review and meta-analysis. Neurosurgery 77:847-874, 2015

12. Kim DY, Lee SH, Chung SK, Lee HY: Comparison of multifidus muscle atrophy and trunk extension muscle strength: percutaneous versus open pedicle screw fixation. Spine (Phila Pa 1976) 30:123-129, 2005

13. Lau D, Chou D: Posterior thoracic corpectomy with cage reconstruction for metastatic spinal tumors: comparing the mini-open approach to the open approach. J Neurosurg Spine 23:217-227, 2015

14. Lau D, Lee JG, Han SJ, Lu DC, Chou D: Complications and perioperative factors associated with learning the technique of minimally invasive transforaminal lumbar interbody fusion (TLIF). J Clin Neurosci 18:624-627, 2011

15. Lau D, Song Y, Guan Z, Sullivan S, La Marca F, Park P: Perioperative characteristics, complications, and outcomes of single-level versus multilevel thoracic corpectomies via modified costotransversectomy approach. Spine (Phila Pa 1976) 38:523-530, 2013

16. Lee JK, Jang JW, Kim TW, Kim TS, Kim SH, Moon SJ: Percutaneous short-segment pedicle screw placement without fusion in the treatment of thoracolumbar burst fractures: is it effective?: comparative study with open short-segment pedicle screw fixation with posterolateral fusion. Acta Neurochir (Wien) 155:2305-23122013

17. Mendoza-Lattes S, Ries Z, Gao Y, Weinstein SL: Proximal junctional kyphosis in adult reconstructive spine surgery results from incomplete restoration of the lumbar lordosis relative to the magnitude of the thoracic kyphosis. Iowa Orthop J 31:199-206, 2011

18. Miyashita T, Ataka H, Kato K, Tanno T: Good clinical outcomes and fusion rate of facet fusion with a percutaneous pedicle screw system for degenerative lumbar spondylolisthesis: minimally invasive evolution of posterolateral fusion. Spine (Phila Pa 1976) 40:E552-E557, 2015

19. Pakzaban P: Modified mini-open transforaminal lumbar interbody fusion: description of surgical technique and assessment of free-hand pedicle screw insertion. Spine (Phila Pa 1976) 41:E1124-E1130, 2016

20. Pingel A, Castein J, Kandziora F: Vertebral body replacement in the thoracolumbar spine via a mini-open, thoracoscopically assisted transthoracic approach. Eur Spine J 24 (Suppl 8):S949-S950, 2015

21. Seng C, Siddiqui MA, Wong KP, Zhang K, Yeo W, Tan SB, et al: Five-year outcomes of minimally invasive versus open transforaminal lumbar interbody fusion: a matched-pair comparison study. Spine (Phila Pa 1976) 38:2049-2055, 2013

22. Shen FH, Marks I, Shaffrey C, Ouellet J, Arlet V: The use of an expandable cage for corpectomy reconstruction of vertebral body tumors through a posterior extracavitary approach: a multicenter consecutive case series of prospectively followed patients. Spine J 8:329-339, 2008

23. Shim JH, Kim WS, Kim JH, Kim DH, Hwang JH, Park CK: Comparison of instrumented posterolateral fusion versus percutaneous pedicle screw fixation combined with anterior 
lumbar interbody fusion in elderly patients with L5-S1 isthmic spondylolisthesis and foraminal stenosis. J Neurosurg Spine 15:311-319, 2011

24. Taghva A, Li KW, Liu JC, Gokaslan ZL, Hsieh PC: Minimally invasive circumferential spinal decompression and stabilization for symptomatic metastatic spine tumor: technical case report. Neurosurgery 66:E620-E622, 2010

25. Uribe JS, Dakwar E, Le TV, Christian G, Serrano S, Smith WD: Minimally invasive surgery treatment for thoracic spine tumor removal: a mini-open, lateral approach. Spine (Phila Pa 1976) 35 (26 Suppl):S347-S354, 2010

26. Wang MY, Bordon G: Mini-open pedicle subtraction osteotomy as a treatment for severe adult spinal deformities: case series with initial clinical and radiographic outcomes. J Neurosurg Spine 24:769-776, 2016

27. Wang MY, Mummaneni PV: Minimally invasive surgery for thoracolumbar spinal deformity: initial clinical experience with clinical and radiographic outcomes. Neurosurg Focus 28(3):E9, 2010

28. Wang MY, Mummaneni PV, Fu KM, Anand N, Okonkwo DO, Kanter AS, et al: Less invasive surgery for treating adult spinal deformities: ceiling effects for deformity correction with 3 different techniques. Neurosurg Focus 36(5):E12, 2014
29. Wang ST, Ma HL, Liu CL, Yu WK, Chang MC, Chen TH: Is fusion necessary for surgically treated burst fractures of the thoracolumbar and lumbar spine?: a prospective, randomized study. Spine (Phila Pa 1976) 31:2646-2653, 2006

\section{Disclosures}

Dr. Chou states that he is a consultant for Globus, Medtronic, and Orthofix, and receives royalties from Globus.

\section{Author Contributions}

Conception and design: both authors. Acquisition of data: Lau. Analysis and interpretation of data: both authors. Drafting the article: Lau. Critically revising the article: both authors. Reviewed submitted version of manuscript: both authors. Approved the final version of the manuscript on behalf of both authors: Chou. Statistical analysis: Lau. Study supervision: Chou.

\section{Correspondence}

Dean Chou, Department of Neurological Surgery, University of California, San Francisco, 505 Parnassus Ave., Box 0112, San Francisco, CA 94143. email: choud@neurosurg.ucsf.edu. 